INPLASY

PROTOCOL

To cite: Yan et al. The efficacy and safety of Tuina for Diabetic Gastroparesis: A protocol for systematic review and meta-analysis. Inplasy protocol 2021110019. doi: 10.37766/inplasy2021.11.0019

Received: 06 November 2021

Published: 06 November 2021

Corresponding author:

Yan Huixin

doctoryhx@126.com

Author Affiliation:

Yueyang Hospital of Integrated

Traditional Chinese and

Western Medicine,Shanghai

University of Traditional

Chinese Medicine.

Support: SMC2013.

Review Stage at time of this submission: Preliminary

searches.

Conflicts of interest:

None declared.

\section{The efficacy and safety of Tuina for Diabetic Gastroparesis: A protocol for systematic review and meta-analysis}

Yan, HX1; An, Y2; Zhang, T3; Zhao, JN4; Yan, JT55.

Review question / Objective: The purpose of this study is to conduct a meta-analysis of Tuina in the treatment of diabetic gastroparesis to clarify its efficacy and safety.

Condition being studied: Diabetic gastroparesis (DGP) is one of the most common complication of diabetes. At present, despite the increasing number of clinical researches of treatment with Tuina have been reported, there are no systematic reviews of Tuina therapy for DGP. Therefore, it is of great significance to evaluate its safety and efficacy as a complementary and alternative therapy.

INPLASY registration number: This protocol was registered with the International Platform of Registered Systematic Review and Meta-Analysis Protocols (INPLASY) on 06 November 2021 and was last updated on 06 November 2021 (registration number INPLASY2021110019).

\section{INTRODUCTION}

Review question / Objective: The purpose of this study is to conduct a meta-analysis of Tuina in the treatment of diabetic gastroparesis to clarify its efficacy and safety.
Condition being studied: Diabetic gastroparesis (DGP) is one of the most common complication of diabetes. At present, despite the increasing number of clinical researches of treatment with Tuina have been reported, there are no systematic reviews of Tuina therapy for DGP. Therefore, it is of great significance to 
evaluate its safety and efficacy as a complementary and alternative therapy.

\section{METHODS}

Participant or population: Patients suffering from diabetic gastroparesis.

Intervention: Simple Tuina or Tuina combined with other ordinary and basic treatment will be the intervention of the experimental group.

Comparator: The control group received treatment with Western medication, Chinese herbal medication, placebo, acupuncture, acupoint injection, and so on, or even with no treatment, will be included.The control group received treatment with Western medication, Chinese herbal medication, placebo, acupuncture, tuina, and so on, or even with no treatment, will be included.

Study designs to be included: This review will include randomized controlled trials (RCTs) on Tuina for DGP published in Chinese and English language.

Eligibility criteria: Studies will include patients diagnosed with DGP, and presenting two or more of the following symptoms: abdominal bloating, pain, belching, nausea, vomiting, loss of appetite, diarrhea, and constipation; The outcomes of related examinations (gastric emptying examination, Bultrasound, endoscopy, and so forth) were delayed gastric emptying and/or retention of food residues.

Information sources: Randomized controlled trials (RCTs) of Tuina therapy in the treatment of DGP will be retrieved from PubMed, Embase, Web of Science, Cochrane Library, China National Knowledge Infrastructure (CNKI), Wan Fang Database (Wang Fang), Chinese Biomedical Literature Database (CBM), VIP Database for Chinese Technical Periodicals (VIP), Medline, and Clinical Trial Register (CTR).We will consider articles published in English or Chinese between database initiation and November 2021.
Main outcome(s): The main outcome of this review is clinical total effective rate.

Additional outcome(s): The additional outcomes include FPG, $2 \mathrm{~h}$ PPG, Traditional Chinese Medicine symptom score, recurrence rate of clinical signs, adverse reactions, etc.

Quality assessment / Risk of bias analysis: Two researchers will use the Cochrane Collaborative tool to independently assess the risk of bias in all final inclusion of trials. Random sequence generation, allocation concealment, blinding of participants and personnel, blindness of outcome assessments, incomplete outcome data, selective reporting, and other bias will be evaluated as low, high, or ambiguous risk in each study. The results of the bias assessment risk will be carefully checked by other researchers, and any disagreement will be finalized after further discussion by all investigators.

Strategy of data synthesis: Review Manager software (Revman, Version 5.3 for Windows) will be used to perform the metaanalysis. The Chi-Squared test and 12 statistic will be used to assess the heterogeneity of literature according to the values of $\mathbf{P}$ and $\mathbf{1 2}$. If the homogeneity is low $(P>0.1 ; 12<50 \%)$, the fixed-effect model will be used for the meta-analysis; otherwise, the random effect model will be selected. However, when the heterogeneity is particularly high, we will first try to find the cause of its occurrence, and then decide the final meta-analysis model to choose.

Subgroup analysis: When there was substantial heterogeneity in the included studies, the subgroup analysis will be conducted according to factors such as condition of disease, course of disease, treatment sites, intervention frequency, age, gender, region, year, sample size, and other factors.

Sensitivity analysis: Sensitivity analysis is also one of the indispensable methods to deal with heterogeneity. The sensitivity analysis, we will conduct, is to explore the 
impact of trial bias risk on preliminary results. If there is significant statistical heterogeneity, sensitivity analysis will exclude low-quality studies, and repeat meta-analysis based on sample size and insufficient data to assess quality and robustness.

Country(ies) involved: China.

Keywords: Tuina, diabetic gastroparesis, systematic review, meta-analysis, protocol.

Contributions of each author:

Author 1 - Yan Huixin.

Email: doctoryhx@126.com

Author 2 - An Yun.

Email: anyun920122@163.com

Author 3 - Zhang Tao.

Email: 510444994@qq.com

Author 4 - Zhao Jiangna.

Email: kfxszjn@163.com

Author 5 - Yan Juntao.

Email: doctoryjt@163.com 